\title{
Trombosis porto-mesentérica aislada secundaria a trauma abdominal cerrado
}

\author{
Isolated porto-mesenteric thrombosis secondary to blunt abdominal trauma
}

\author{
Juan Luis Vélez-Leal', Leidy Carlina López-Vélez²
}

Médico, residente de Medicina de Urgencias, Universidad CES, Medellín, Colombia
Médica general, Servicio de Oncología, Hospital Pablo Tobón Uribe, Medellín, Colombia

\section{Resumen}

En este artículo se revisan los aspectos más importantes de la epidemiología, fisiopatología, abordaje diagnóstico y terapéutico de la trombosis del sistema venoso portal y mesentérico, para finalizar discutiendo la experiencia clínica de un caso a la luz de la literatura relevante. Esta complicación es usualmente relacionada con afecciones sistémicas y hepáticas, pero rara vez se presenta aislada y relacionada con el trauma. En ausencia de tratamiento adecuado durante la etapa aguda, la trombosis puede evolucionar hacia el infarto intestinal y la muerte del paciente. En casos crónicos, se ha asociado con la degeneración del sistema venoso portal hacia cambios por hipertensión portal crónica.

Palabras clave: traumatismos abdominales; traumatismos cerrados; trombosis de la vena; isquemia mesentérica; sistema porta.

\begin{abstract}
This article reviews the epidemiology, pathophysiology, diagnostic and therapeutic approach of the portal and mesenteric venous system thrombosis, with a clinical case discussion considering the relevant literature. This complication is usually related to systemic and hepatic conditions, but rarely occurs isolated and related to trauma. In the absence of adequate treatment during the acute stage, thrombosis can progress to intestinal infarction and death. In chronic cases, it has been associated with degeneration of the portal venous system into changes due to chronic portal hypertension.
\end{abstract}

Keywords: abdominal injuries; blunt injuries; venous thrombosis; mesenteric ischemia; portal system.

Fecha de recibido: 08/11/2019 - Fecha de aceptación: 28/05/2020

Correspondencia: Juan Luis Vélez Leal, Calle 78B \# 69-240, Medellín, Colombia. Teléfono: 57 (4) 4459000 Ext 9225.

Correo electrónico: velezl.juan@gmail.com

Citar como: Vélez-Leal JL, López-Vélez LC. Trombosis porto-mesentérica aislada secundaria a trauma abdominal cerrado. Rev Colomb Cir. 2020;35:675-81. https://doi.org/10.30944/20117582.497

Este es un artículo de acceso abierto bajo una Licencia Creative Commons - BY-NC-ND https://creativecommons.org/licenses/by-ncnd/4.0/deed.es 


\section{Introducción}

No hay duda de que el trauma es un problema de salud pública en Colombia: solo los accidentes de tránsito respondieron por el $28,4 \%$ de las muertes violentas y el II,I \% de las lesiones no fatales durante el primer trimestre de $202 \mathrm{O}^{\mathrm{I}}$. Por lo anterior, el personal médico debe mantenerse actualizado en la atención urgente del trauma y de sus complicaciones. Como lo demuestra el caso a discutir, existen diagnósticos potencialmente mortales tan infrecuentes que pocas veces se sospechan y por ello rara vez son diagnosticados. Entre estas complicaciones se incluye la trombosis venosa portal y mesentérica (TVPM) aislada secundaria a trauma abdominal cerrado.

\section{Caso clínico}

Se trata de un paciente en la cuarta década de la vida, previamente sano, que sufre trauma toracoabdominal cerrado en un accidente de tránsito de alta cinemática, en calidad de conductor de automóvil. Refiere traumatismo sobre la reja costal anterior y el abdomen, al golpearse contra el volante y la bolsa de aire. No hay extricación ni muerte en la escena. Acude a urgencias por sus propios medios y en una primera atención ingresa a observación, se brinda analgesia, se realizan gases arteriales y radiografías del tórax. Ante la ausencia de alteraciones paraclínicas y adecuado estado clínico, sin dolor abdominal, se decide dar egreso con analgesia oral, incapacidad laboral y signos de alarma para consultar de nuevo.

El paciente ingresa por segunda vez al servicio de urgencias diez días después del accidente, refiriendo cuatro días de náuseas constantes, distensión abdominal, dolor tipo cólico en epigastrio e hipocondrio derecho, de predominio postprandial, gravativo y que empeora con los cambios de posición en el decúbito. $\mathrm{Al}$ examen físico sus signos vitales eran normales, no se evidenciaban estigmas de trauma toracoabdominal pero la palpación del abdomen superior era notablemente dolorosa, por lo que se decidió realizar una tomografía computarizada (TC) de abdomen total con contraste endovenoso. En la misma se documenta trombosis de la vena me- sentérica superior y de la rama distal de la porta derecha, sin evidencia de lesiones traumáticas en órganos sólidos ni vísceras huecas (figura I).

El paciente es hospitalizado, se inicia anticoagulación con heparinas de bajo peso molecular por vía subcutánea (enoxaparina $\mathrm{Img} / \mathrm{kg}$ cada I2 horas) y Warfarina. Se estudia a profundidad para autoinmunidad y trombofilias, descartándose causas primarias de la trombosis (tabla I). También se indagó por consumo de productos naturistas, anabólicos u otros suplementos, para los que el paciente negó consumo. Con el inicio de la terapia médica presenta rápida mejoría de los síntomas y una semana después es dado de alta para continuar terapia anticoagulante oral. Desafortunadamente el paciente no asiste a los controles ambulatorios y no se conoce información clínica de su evolución.

\section{Revisión de tema y discusión}

La trombosis del sistema venoso portal (TVPM), que comprende las venas porta, esplénica y mesentéricas, es una complicación usualmente relacionada con afecciones sistémicas y hepáticas.

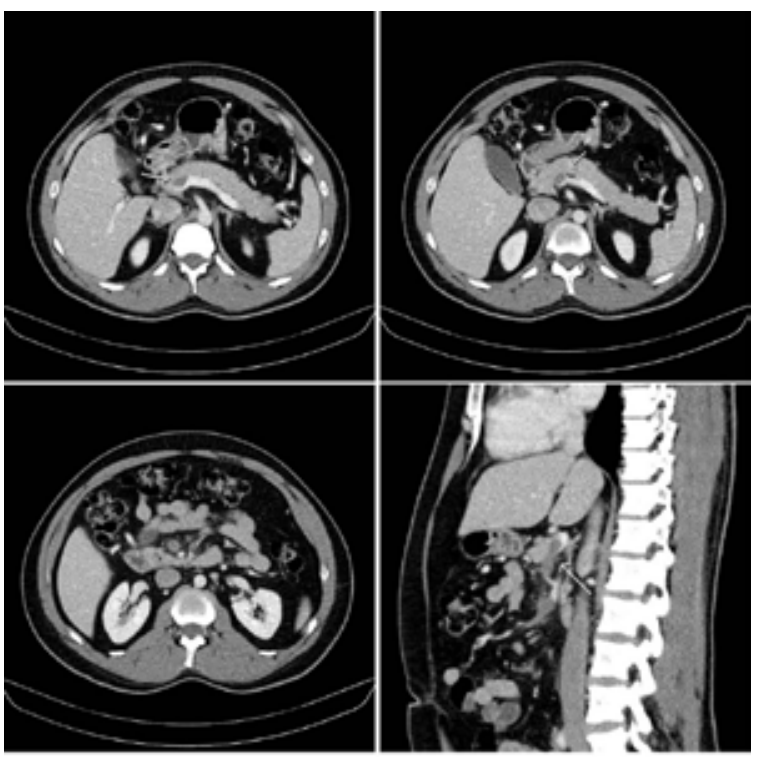

Figura 1. Estriación de la grasa mesentérica rodeando vasos mesentéricos superiores, con trombosis de la vena mesentérica superior y trombosis de la rama distal de la porta derecha en su rama anterosuperior. 
Tabla 1. Estudios de laboratorio clínico practicados al paciente

\begin{tabular}{llll}
\hline \multicolumn{2}{c}{ Estudios de extensión realizados } \\
\hline Alanina aminotransferasa (ALT) & $28 \mathrm{U} / \mathrm{l}$ & ELISA VIH & No reactivo \\
\hline Aspartato aminotransferasa (AST) & $19 \mathrm{U} / \mathrm{l}$ & VDRL & No reactivo \\
\hline Fosfatasa Alcalina & $97 \mathrm{U} / \mathrm{l}$ & Mutación GFII & $\begin{array}{l}\text { Homocigoto normal, } \\
\text { mutación G20210A negativo }\end{array}$ \\
\hline Bilirrubina Total / Bilirrubina Directa & $0,4 / 0,16 \mathrm{mg} / \mathrm{dl}$ & F-V Leiden & $\begin{array}{l}\text { Homocigoto normal, } \\
\text { mutación G1691A negativo }\end{array}$ \\
\hline Creatinina & $1,09 \mathrm{mg} / \mathrm{dl}$ & Homocisteína & 9,3 umol/L \\
\hline Nitrógeno ureico en sangre (BUN) & $12,2 \mathrm{mg} / \mathrm{dl}$ & Anti CL IgG e IgM & Negativo \\
\hline Serie roja & $13,7 \mathrm{~g} / \mathrm{dl}$ & B2- GP IgG e IgM & Negativo \\
\hline Serie blanca & $\begin{array}{l}9.800 \text { leucocitos / } 62,1 \% \\
\text { neutrófilos }\end{array}$ & ANAs & $1: 80$ patrón moteado \\
\hline $\begin{array}{l}\text { Tiempo parcial de tromboplastina } \\
\text { (TPT) / INR }\end{array}$ & $30,2 \mathrm{seg} / 1,08$ & Actividad de proteínas C y S & Se difieren por cuadro agudo \\
\hline Tiempo de protrombina (TP) & $12,3 \mathrm{seg}$ & Citoquímico de orina & $\begin{array}{l}\text { Proteínas (+), sin hematuria } \\
\text { ni piuria }\end{array}$ \\
\hline
\end{tabular}

* ELISA: ensayo inmunológico de cuarta generación, VDRL: prueba no treponémica, Mutación GFII: detección de la mutación del gen de protrombina, F-V Leiden: factor V Leiden, Anti-CL: anti cardiolipinas, B2-GP: beta 2 glicoproteínas, ANAs: anticuerpos antinucleares

Su relación con el trauma de abdomen cerrado es un verdadero desafío diagnóstico, dada su ocurrencia esporádica y presentación clínica inespecífica $^{2-5}$. En una revisión reciente realizada por Nishimura et al., ${ }^{6}$ se indican 19 casos reportados en Pubmed relacionados con trauma abdominal, en su mayoría secundarios a eventos de alta cinemática y con lesión concomitante de órganos abdominales. La aparición aislada de TVPM se ha tomado como un diagnóstico raro y de exclusión, resaltando lo inusual del caso reportado ${ }^{2,7-9}$.

\section{Epidemiología}

El riesgo de sufrir de TVPM para la población sana es del I \%, mientras que en presencia de factores de riesgo es del orden de 4,4 al $15 \%{ }^{6,10}$. Esta patología representa el o,or \% de los ingresos hospitalarios por urgencias quirúrgicas ${ }^{3,4,11} \mathrm{y}$ el o,002 \% al o,06 \% de los ingresos totales ${ }^{12}$. La trombosis venosa mesentérica aguda ocurre con mayor frecuencia en el íleon (64-83\%) y yeyuno (50-8I \%), seguido del colon (I4 \%) y duodeno $(4-8 \%)$.

En cuanto al pronóstico, la TVPM responde por cerca del $2 \%$ al $15 \%{ }^{4, I, 13}$ de los casos de isquemia mesentérica aguda, y acarrea una menor tasa de mortalidad que su contraparte arterial (44\% vs. $89 \%)^{12}$. El mayor determinante de un desenlace adverso es la presencia de infarto intestinal, situación ahora infrecuente gracias al advenimiento de los métodos diagnósticos modernos y las terapias anticoagulantes que han permitido disminuir la mortalidad asociada en las últimas cuatro décadas ${ }^{12,14}$.

\section{Presentación clínica}

El síntoma más frecuentemente asociado es el dolor abdominal, que ocurre en un $90 \%$ a 100 $\%$ de los casos $^{3,4,14,15}$. Usualmente se localiza en epigastrio y mesogastrio, es gravativo, sus características son inespecíficas y se exacerba con la ingesta de alimentos. Es común encontrar disociación entre la magnitud del dolor descrito y los hallazgos al examen físico $3,4,10,12,5,16$. Se han descrito otros síntomas como pérdida de peso, constipación, dispepsia, distensión, diarrea y melenas. Se ha reportado que hasta el $75 \%$ de los pacientes consultarán pasadas las 48 horas de inicio de los síntomas y que el tiempo transcurrido hasta el diagnóstico es un factor predictivo del desenlace y la necesidad de cirugía ${ }^{4,14}$.

En el caso reportado, se llegó al diagnóstico tardío gracias a la alta sospecha de lesiones intrabdominales, a partir del examen físico dis- 
cordante con la sintomatología del paciente, y del conocimiento de la cinemática del trauma previo. La presentación clínica de la TVPM asociada al trauma difiere con respecto a la secundaria a causas sistémicas en que la primera tiene manifestaciones menos ominosas, más larvadas y localizadas, dada la menor carga trombótica ${ }^{2}$. Además, las lesiones extra abdominales asociadas al trauma pueden ser distractores en la evaluación clínica inicial.

\section{Fisiopatología}

La TVPM tradicionalmente se ha asociado a patologías propias del hígado, estados inflamatorios intrabdominales y retroperitoneales, neoplasias y estados protrombóticos hereditarios ${ }^{4,6-8,1,1,1,14,16,17}$. Se describe que hasta el $60 \%$ de los pacientes que sufren de TVPM presentan alguna trombofilia de forma concomitante ${ }^{6}$ y que del $20 \%$ al $40 \%$ tienen el antecedente de haber presentado trombosis venosa profunda ${ }^{12}$. No obstante, del 2I \% al $49 \%$ de todos los casos resultan idiopáticos $^{3,12}$ (tabla 2).

En cuanto a la fisiopatología de la TVPM traumática, se propone que el trauma genera daño a través de las fuerzas de cizallamiento que lesionan el endotelio e inician la cascada trombótica. La patología hepatobiliar subyacente agrava el cuadro a través de la estasis venosa. En adición, un estado protrombótico sistémico, aumenta el riesgo de formación y persistencia de trombosis ${ }^{2,8}$.

Se considera que en el caso descrito, la presentación tardía del dolor abdominal es explicada por la propagación paulatina de la trombosis, iniciada por lesiones al endotelio, secundarias a las fuerzas de compresión ocurridas al haberse golpeado sobre el área toracoabdominal con la bolsa de aire del vehículo ${ }^{2,8}$.

Es difícil predecir cuán pronto ocurrirá un infarto intestinal, con rangos reportados desde horas hasta cinco semanas ${ }^{2}$. Este tiempo comprende el lapso en el que la trombosis progresa hasta los arcos venosos y vasos rectos intestinales, ocluyendo completamente el retorno venoso ${ }^{12}$. Un factor que se ha asociado a la isquemia e infarto intestinal es la asociación de vasoespasmo arterial, que incluso puede persistir cuando la trombosis venosa ha resuelto ${ }^{12}$. La aparición de signos de irritación peritoneal, hematemesis y hematoquecia, son considerados un indicador de mal pronóstico y de progresión a isquemia transmural ${ }^{3, \mathrm{III}, 12}$.

No existe un consenso para diferenciar el cuadro agudo del crónico. Se han tomado los cambios de degeneración cavernomatosa de la vena porta, los signos clínicos de hipertensión portal y la esplenomegalia como marcadores de la afección crónica ${ }^{13,55,17}$.

\section{Diagnóstico}

Actualmente se considera la TC con contraste venoso como el método diagnóstico de elección, debido a sus costos más bajos en comparación con la resonancia magnética, su amplia disponibilidad y su excelente sensibilidad ${ }^{3-5,13}$. Los hallazgos tomográficos incluyen asas intestinales

Tabla 2. Etiologías de la trombosis del sistema venoso portal

\begin{tabular}{ll}
\hline Primarias & Deficiencia de proteína C y S \\
& Deficiencia de antitrombina III \\
& Mutación del factor V Leiden \\
& Mutación de protrombina del gen 20210 A \\
& Hiperhomocisteinemia \\
& Resistencia a la proteína C activada \\
& Anticoagulantes lúpicos \\
& Anticuerpos antifosfolípidos \\
& Anemia de células falciformes \\
\hline Secundarias & Enfermedades mieloproliferativas \\
& Neoplasias intrabdominales \\
& Pancreatitis \\
& Enfermedad inflamatoria intestinal \\
& Embarazo \\
Condiciones posoperatorias (esplenectomía, \\
colectomía) \\
Cirrosis \\
Hipertensión portal \\
Síndrome nefrótico \\
Politraumatismo \\
Trauma abdominal penetrante \\
Trauma abdominal cerrado \\
\hline Idiopáticas \\
Consumo de productos naturistas \\
Uso de anticonceptivos orales
\end{tabular}


dilatadas y engrosadas, estriación de la grasa mesentérica, ascitis, un halo o aspecto blanco del intestino, defectos de llenado de los vasos y neumatosis intestinal ${ }^{3,5,15}$. La TC presenta como ventajas el no ser operador dependiente, la posibilidad de identificar otras lesiones asociadas, así como la extensión exacta de la trombosis, y detectar signos de isquemia intestinal ${ }^{8,18}$.

Aunque la venografía por resonancia magnética ofrece una mejor visualización de la anatomía y las posibles causas subyacentes ${ }^{3,5,15}$, su falta de disponibilidad hace de la TC el estudio preferido en los escenarios agudos ${ }^{3}$. Por su parte, la ecografía puede perfilarse como una modalidad de estudio primaria para el diagnóstico por fuera del escenario del trauma y en el seguimiento ${ }^{3,5}$.

La realización de la TC abdominal con contraste venoso en el caso discutido se considera acertada pues este es el estándar de oro para el diagnóstico de TVPM4 . Lo anterior permitió identificar la etiología del dolor abdominal y el inicio oportuno de la terapia anticoagulante.

Completando los estudios imagenológicos, se puede considerar la angiografía en aquellos pacientes que son candidatos a terapia intraarterial y para el estudio de síntomas abdominales continuos o nuevos en pacientes con trombofilia conocida, cuya TC contrastada fue normal o equívoca $^{5,12,15}$. Finalmente, la laparotomía se ha tomado como modalidad diagnóstica en algunas series de casos, pero actualmente su principal indicación es la sospecha de infarto intestinal ${ }^{12,13}$.

\section{Tratamiento}

El manejo actualmente propuesto incluye la anticoagulación con heparinas no fraccionadas o de bajo peso molecular, con transición posterior a antagonistas orales de la vitamina $K$, para un objetivo de INR de 2 a $3^{6,13,15}$. Lo anterior ha demostrado un aumento en la supervivencia y disminución de la recurrencia ${ }^{13}$. En ausencia de una trombofilia conocida, se recomienda anticoagulación por un tiempo entre 6 y I2 meses ${ }^{\mathrm{II}, \mathrm{I} 5}$; sin embargo, en pacientes con causas rápidamente reversibles tales como un trauma, se recomien- da una duración 3 a 6 meses ${ }^{4,18}$. A la fecha, no existe una recomendación sobre el método de anticoagulación, ni la duración de esta, que sea sustentada con evidencia fuerte ${ }^{3, \mathrm{II}}$.

Si bien la literatura describe otras medidas coadyuvantes en el tratamiento del episodio agudo, como la reanimación hídrica, reposo intestinal, e inclusive el inicio temprano de terapia antibiótica profiláctica para limitar la translocación bacteriana ${ }^{4,13}$, en el paciente presentado únicamente se instauró analgesia por horario y de rescate, de forma adicional a los anticoagulantes, resultando en un desenlace temprano favorable.

La necesidad de iniciar terapia antitrombótica y vigilancia hospitalaria se justifica en el riesgo de progresión de la enfermedad y de isquemia mesentérica ${ }^{3}$. El objetivo de la anticoagulación es evitar el infarto intestinal previniendo la extensión de la trombosis y generando recanalización venosa $^{15,18}$. Es difícil calificar lo apropiado de esta intervención clínica dado que las recomendaciones de manejo se basan en consensos de expertos o resultados clínicos en series de $\operatorname{casos}^{\mathrm{I}}$. En cualquier caso, se desincentiva el uso de agentes trombolíticos dado el riesgo de hemorragia y la baja tasa de éxito"

Los procedimientos quirúrgicos terapéuticos incluyen desde la laparotomía exploratoria hasta la resección intestinal y la trombectomía ${ }^{13}$. En aproximadamente el Io \% de los casos, la TVPM no responde adecuadamente a los anticoagulantes y pudiera requerirse la trombectomía quirúrgica o la cirugía de derivación, considerando la complejidad de los procedimientos y que el éxito de la recanalización es cercano al $30 \%{ }^{6}$.

En cuanto al seguimiento, se espera que la recanalización de la vena porta ocurra en seis meses, mientras que para las venas mesentérica y esplénica ocurre hacia los doce meses. Más de la mitad de los pacientes que no logran la recanalización desarrollarán varices esofágicas, con probabilidades de presentar en los siguientes dos años, sangrado variceal y ascitis en el I2 \% y I6 $\%$, respectivamente ${ }^{15}$. Desafortunadamente el paciente no acudió al seguimiento ambulatorio 
y se desconoce si presentó alguna complicación o signos de TVPM crónica.

\section{Conclusiones}

La TVPM es una patología vascular gastrointestinal grave que, en ausencia de tratamiento, puede llevar a isquemia intestinal secundaria y a las complicaciones tradicionalmente descritas para el síndrome de hipertensión portal crónica ${ }^{4,8,9,13,15}$. Su presentación aislada y en relación con trauma cerrado de abdomen es sumamente infrecuente. Se sugiere abordar cada caso de manera individual, considerando las variables del contexto clínico específico y la necesidad del tratamiento intrahospitalario en un centro de alta complejidad, que garantice el abordaje interdisciplinario entre los equipos quirúrgicos y clínicos, permitiendo que se estudien a fondo las causas primarias de trombosis, al tiempo que se continua la terapia antitrombótica y se considera la consulta quirúrgica ante la sospecha de infarto intestinal, sobre todo en pacientes con persistencia de dolor intenso, sangrado rectal, ascitis o evidencia de disfunción orgánica ${ }^{15}$.

Agradecimientos: los autores agradecen al Hospital Pablo Tobón Uribe y en especial al servicio de urgencias adultos por incentivar continuamente la atención con altos estándares humanos y científicos.

\section{Consideraciones éticas}

Consentimiento informado: Este estudio es una revisión de la literatura, y como tal no hay necesidad de un consentimiento informado. Se obtuvo el consentimiento informado por parte del paciente para la utilización de su información clínica e imágenes. El Comité de Investigaciones y Ética en investigaciones Institucional aprobó la realización del estudio

Conflicto de intereses: ninguno de los autores presenta conflicto de intereses.

Fuente de financiación: recursos propios de los autores.

\section{Referencias}

I. Instituto Nacional de Medicina Legal y Ciencias Forenses, Colombia. Boletín estadístico mensual marzo de 2020. Subdirección de servicios forenses. Centro de Referencia Nacional sobre Violencia. Fecha de consulta: I2 de mayo de 2020. Disponible en:

https://www.medicinalegal.gov.co/documents/ 20I43/ 494197/Boletin+marzo+2020.pdf/ce533028-7683-6Ie2e95d-37011224c7db

2. McCune WS, Keshishian JM, Blades BB. Mesenteric thrombosis following blunt abdominal trauma. Ann Surg. 1952;135:606-I4.

https://doi.org/I0.I097/00000658-195205000-00004

3. Smith ME, Percy K, Ritz RB. Acute superior mesenteric venous thrombosis in a young patient without risk factors. Emerg Med. 2018;50:199-202.

https://doi.org/Io.I2788/emed.20I8.oIo8

4. Garaci FG, Vasili E, Bocchinfuso F, Lacchè A, Floris R. Post-traumatic superior mesenteric venous thrombosis with subsequent extension to the portal vein. Radiol Case Reports. 20I9;I4:I5I-5.

https://doi.org/IO.IOI6/j.radcr.20I8.Io.oI4

5. Hauenstein K, Li Y. Radiological diagnosis of portal/mesenteric vein occlusion. Viszeralmedizin. 20I4;30:382-7. https://doi.org/I0.II59/000370055

6. Nishimura E, Misawa T, Kitamura H, Fujioka S, Akiba T, Yanaga K. A case of portal vein thrombosis caused by blunt abdominal trauma in a patient with low protein C activity. Clin J Gastroenterol. 20I8;II:507-I3. https://doi.org/IO.I007/sI2328-0I8-0879-8

7. Rajkomar V, Kyerematen E, Mysore P, Penston J. Thrombosis of the portal venous system following blunt abdominal trauma. BMJ Case Rep. 2010:2-5. https://doi.org/IO.II36/bcr.II.2009.2429

8. Harnik IG, Brandt LJ. Mesenteric venous thrombosis. Vasc Med. 20I0;15:407-I8. https://doi.org/IO.II77/1358863XI0379673.

9. Gopal SV, Smith I, Malka V. Acute portal venous thrombosis after blunt abdominal trauma. Am J Emerg Med. 2009;27:372.eI-372.e3. https://doi.org/Io.IoI6/j.ajem.2008.07.02I

Io. Sogaard KK, Astrup LB, Vilstrup H, Gronbaek H. Portal vein thrombosis; risk factors, clinical presentation and treatment. BMC Gastroenterol. 2007;7:I-6. https://doi.org/IO.II86/I47I-230X-7-34

II. Lim KH, Jang J, Yoon HY, Park J. Acute superior mesenteric vein thrombosis associated with abdominal trauma. A rare case report and literature review. Medicine (Baltimore). 20I7;96:e8863. https://doi.org/I0.I097/MD.000ooooooooo8863

I2. Blumberg SN, Maldonado TS. Mesenteric venous thrombosis. J Vasc Surg Venous Lymphat Disord. 2016;4:50I-507. https://doi.org/IO.IOI6/j.jvsv.2016.04.002 
I3. Lang SA, Loss M, Wohlgemuth WA, Schlitt HJ. Clinical management of acute portal/mesenteric vein thrombosis. Viszeralmedizin. 2014;30:394-400. https://doi.org/IO.II59/000369896

I4. Yanar F, Agcaoglu O, Gok AFK, Sarıcı IS, Ozçınar B, Aksakal N, et al. The management of mesenteric vein thrombosis: A single institution experience. Turkish J Trauma Emerg Surg. 2013;19:223-8. https://doi.org/I0.5505/tjtes.2013.47542

I5. Garcia-Pagán JC, Buscarini E, Janssen HLA, Leebeck FWI, Plessier AI, Rubbia-Brandt, et al. European Association for the Study of the Liver Clinical Practice Guidelines: Vascular diseases of the liver. J Hepatol. 2016;64:I79-202.

https://doi.org/IO.IOI6/j.jhep.2015.07.040
I6. Harward TRS, Green D, Bergan JJ, Rizzo RJ, Yao JST. Mesenteric venous thrombosis. J Endovasc Surg. I989;9:328-33.

https://doi.org/I0.IOI6/074I-52I4(89)90053-0

I7. Hall TC, Garcea G, Metcalfe M, Bilku D, Dennison AR. Management of acute non-cirrhotic and non-malignant portal vein thrombosis: A systematic review. World J Surg. 20II;35:2510-20.

https://doi.org/IO.IOO7/s00268-OII-II98-O

I8. Sarmiento-Burbano WA, Otero-Regino W, Parga-Bermúdez JE. Trombosis venosa portal posesplenectomía por anemia hemolítica. Reporte de caso y revisión de la literatura. Rev Colomb Gastroenterol. 2019;34:9I-6. https://doi.org/I0.22516/25007440.358 